\title{
Application of Low Temperature Broad Ion Milling for Crystallographic Orientation Analysis of Biocalcite
}

\author{
Jamil J. Clarke
}

Hitachi High Technologies America, Inc., Nanotechnology Systems Division, 944 Clopper Rd, Gaithersburg, Maryland 20878 USA

Nano-Physics science and the technologies developing for sub-micron analysis are ever-increasing in both academic and industrial research \& development fields. It has been understood for several years that scanning electron microscopy allows for measurable observations and analysis at levels in the micron and nanometer range. The need for damage-less observation for fine structures still remain an important factor for the examination of soft and brittle materials. Site specificity at the nanometer level is required to investigate crystal orientation in order to develop materials with characteristics favorable for industrial purposes such as improving strength and durability in consumer end products [1]. Soft materials such as calcite have become a primary interest for SEM imaging and analysis. Conventional sample preparation techniques often require several polishing steps to produce a smooth surface prior to EBSP analysis. Broad ion milling using a Hitachi ion milling system, model IM4000 with a cold block attachment can aid to reduce steps required for preparing soft materials mechanically. The IM4000 makes it possible to create a cross-section without mechanical artifacts. Temperature sensitive materials are subject to thermal deformation during the ion milling process; therefore with the advent of low temperature cooling, thermally sensitive materials such as calcite can be performed with successful results.

Figure 1shows the area along the barnacle's baseplate of interest, for cross-section observation. The sample barnacle was halved and mounted with epoxy. $\mathrm{M}$ echanical polishing was performed for resurfacing the cross-section face of the baseplate. The sample and cross-section stage equipped with a cold block was placed in a freezer for roughly 3 hours in preparation for low temperature broad ion milling. Figure 2 shows the result by SEM imaging of mechanical preparation of the baseplate crosssection and of the same area low temperature broad ion milled. Calcite grain contrast is observable along the baseplate indicating a high quality cross-section face that can be used for EBSP analysis. An area of the broad ion milled base plate cross-section was selected for EBSP analysis to demonstrate the practicality of low temperature broad ion milling as a viable method for preparing calcite based biomaterials or other calcitic materials. Figure 3 shows the resulting EBSP analysis of the barnacle baseplate after low temperature broad ion milling at an acceleration voltage of $3 \mathrm{kV}$ utilizing a cold block to alleviate thermal deformation. This study emphasized that through low temperature broad ion beam technology, the fine surface calcite crystals along the base plate can be achieved with a defect free crosssection without inducing mechanical or thermal stress in the soft material. The effectiveness of utilizing the IM4000 equipped with a cold block has aided to reduce steps required for preparing soft materials mechanically.

References:

[1] Goetz, A. J. and Steinmetz, D. R., et al., Acta Biomaterialia v7 (2011), Interdigitating biocalcite dendrites form a 3-D jigsaw structure in brachiopod shells, p. 2237-2243. 
[2] Hui, Chung-Yuen, et al., J.R. Soc (2011), Barnacles Resist Removal by Crack Trapping, doi:10.1098/rsif.2010.0567

[3] The author acknowledges Alexis C. Lewis Ph.D., Naval Research Laboratory, D.C., USA for the barnacle baseplate material used in this study.

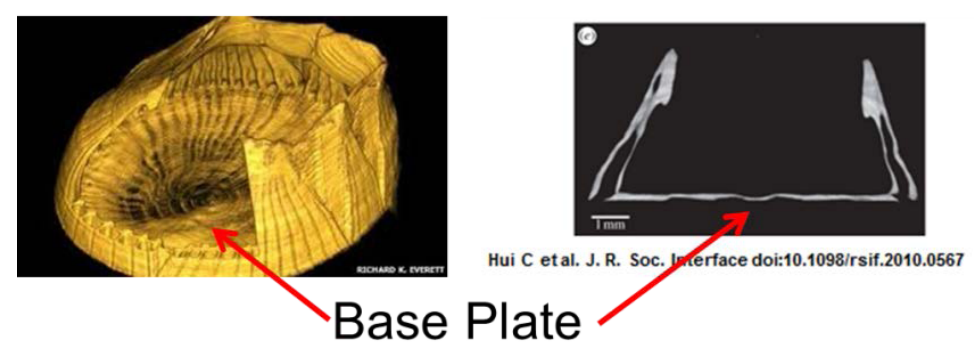

Figure 1. X-ray and tomogram image examples shown of area of interest to be prepared by ion milling along the baseplate [2].
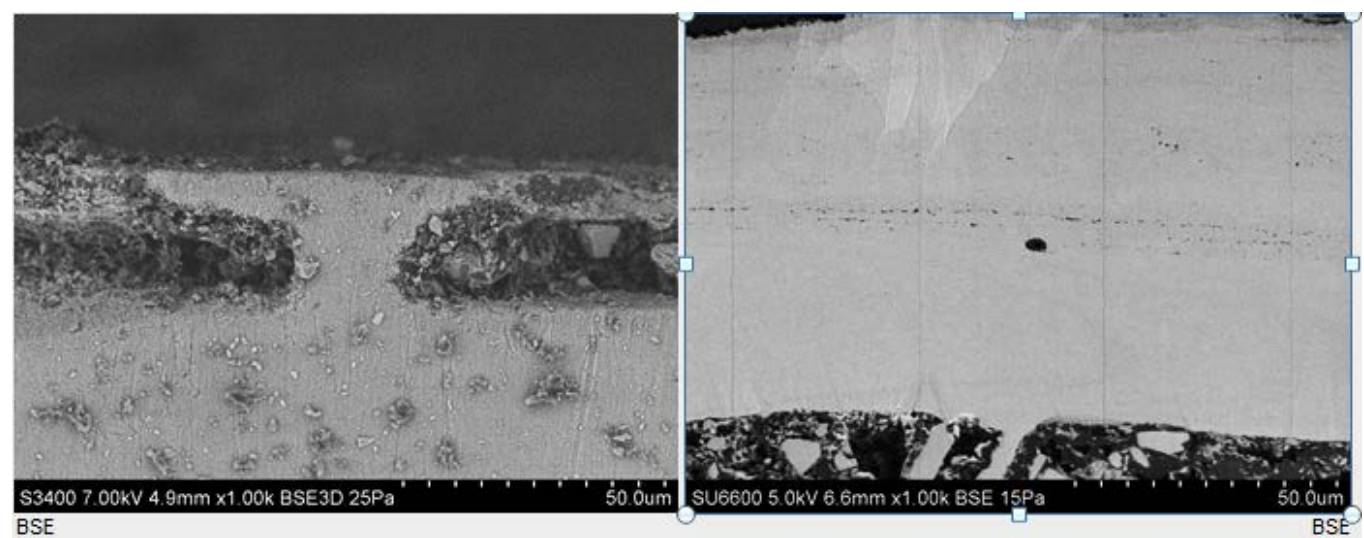

Figure 2. Barnacle baseplate preparation by mechanical polishing (Left) and ion milling (right).

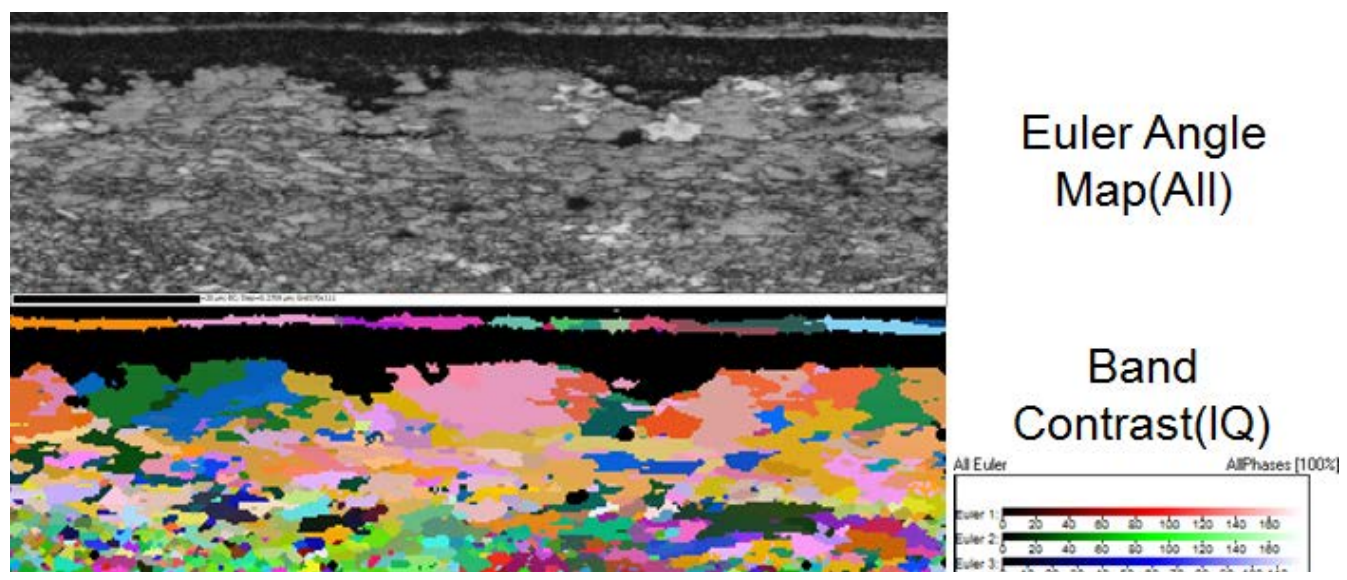

Figure 3. EBSP map quality and Euler angle map along the barnacle baseplate. 